\title{
Characterization of P-Compactly Packed Modules
}

\author{
Lamis J. M. Abu Lebda
}

\begin{abstract}
Let $\mathrm{R}$ be a commutative ring with 1 , and $\mathrm{M}$ is a (left) $\mathrm{R}$-module. We introduce the concepts of (strongly) pcompactly packed submodules as: A proper submodule $N$ of an R-module $M$ is called P-Compactly Packed if for each family $\left\{N_{\alpha}\right\}_{\alpha \in \Lambda}$ of primary submodules of $M$ with $N \subseteq \cup_{\alpha \in \Lambda} N_{\alpha}$, there exists $\alpha_{1}, \alpha_{2}, \ldots, \alpha_{n} \in \Lambda$ such that $N \subseteq \bigcup_{i=1}^{n} N_{\alpha_{i}}$. If $N \subseteq N_{\beta}$ for some $\beta \in \Lambda$, then $N$ is called Strongly P-Compactly Packed. In this paper, we list some basic properties of this concept. In addition, the necessary and sufficient conditions for an $R$-module $M$ to be (strongly) P-Compactly Packed are investigated.We also generalize the Prime Avoidance Theorem for modules that was proved in [7] to the Primary Avoidance Theorem for modules. Furthermore, we find the conditions on an R-module $M$ that make the following important result true, that is for a multiplication Bezout module $M, M$ is strongly $P$ - compactly packed if and only if every primary submodule of $M$ is strongly P- compactly packed.
\end{abstract}

Index Terms-P-compactly packed submodule, Strongly pcompactly packed submodule, MAXIMAL submodule, bezout module.

\section{INTRODUCTION}

Zaynab A.A.Al-Ani generalized the concept of compactly packed rings to modules and introduced the definition of compactly packed submodule and strongly compactly packed submodule; a proper submodule $\mathrm{N}$ of an R-module $\mathrm{M}$ is called compactly Packed if for each family $\left\{N_{\alpha}\right\}_{\alpha \in \Lambda}$ of prime submodules of $M$ with

$N \subseteq \bigcup_{\alpha \in \Lambda} N_{\alpha} \quad$ there exist $\alpha_{1}, \alpha_{2}, \ldots, \alpha_{n} \in \Lambda$ such that $N \subseteq \bigcup_{i=1}^{n} N_{\alpha_{i}}$. If $N \subseteq N_{\beta}$ for some $\beta \in \Lambda$ then $N$ is called Strongly Compactly Packed. A module $M$ is said to be Compactly Packed (Strongly Compactly Packed) if every proper submodule of $\mathrm{M}$ is compactly packed (or strongly compactly packed) submodule [1].

In this paper, we discuss the situation when the union of a family of primary submodules of $\mathrm{M}$ is considered.

C. P. Lu generalized the Prime Avoidance Theorem to modules in terms of prime submodules [5]. We consider a generalization of this theorem to modules in terms of primary submodules.

\section{P-COMPACtly PACKed AND StRONGLY P- COMPACTLY PACKED SUBMOdULES}

We introduce the following definition for p-compactly packed submodule and strongly p-compactly packed

Manuscript received May 10, 2012; revised June 12, 2012

L. J. M. A. Lebda is with the Abu Dhabi University, UAE (e-mail: lamis_jomah@yahoo.com). submodule.

\section{Definition}

A proper submodule $\mathrm{N}$ of an $\mathrm{R}$-module $\mathrm{M}$ is called $\mathrm{P}$ Compactly Packed if for each family $\left\{N_{\alpha}\right\}_{\alpha \in \Lambda}$ of

primary submodules of $M$ with $N \subseteq \bigcup_{\alpha \in \Lambda} N_{\alpha}$, there exist $\alpha_{1}, \alpha_{2}, \ldots, \alpha_{n} \in \Lambda$ such that $N \subseteq \bigcup_{i=1}^{n} N_{\alpha_{i}}$.

If $N \subseteq N_{\beta}$ for some $\beta \in \Lambda$, then $N$ is called Strongly PCompactly Packed.

A module $\mathrm{M}$ is said to be P-Compactly Packed (Strongly P-Compactly Packed) if every proper submodule of $\mathrm{M}$ is $\mathrm{p}$ compactly packed (strongly p-compactly packed).

It is clear every strongly p-compactly packed submodule is a p-compactly packed submodule but the converse is not true is general, as is seen by the following example.

\section{Example}

Let $V$ be a vector space of dimension greater than 2 over the field $F=Z / 2 Z$. Then every subspace of $V$ is prime, so every subspace of $V$ is primary. Let $e_{1}$ and $e_{2}$ be distinct vectors of a basis for $V, \quad V_{1}=e_{1} F$, $V_{2}=e_{2} F, \quad V_{3}=\left(e_{1}+e_{2}\right) F, \quad$ and $L=\left\{0, e_{1}, e_{2}, e_{1}+e_{2}\right\}=V_{1} \cup V_{2} \cup V_{3}$ is an efficient union of three primary submodules with $\sqrt{\left[V_{i}: M\right]}=(0)$, but $L \not \subset V_{i}$ for every $i=1,2,3$.

In the following we give a condition under which the converse holed. For that we give a generalization of the prime avoidance theorem [5] in terms of primary submodules.

\section{Definition}

Let $L_{1}, L_{2}, \ldots, L_{n}$ be submodules of an R-module $\mathrm{M}$. We call a covering $L \subseteq L_{1} \cup L_{2} \cup \ldots \cup L_{n}$ efficient if no $L_{k}$ is superflous. Analogously we shall say $L=L_{1} \cup L_{2} \cup \ldots \cup L_{n}$ is an efficient union if none of the $L_{k}{ }^{\prime} s$ may be excluded.

Any cover or union consisting of submodules of $\mathrm{M}$ can be reduced to an efficient one called an efficient reduction by deleting any unnecessary submodules. A covering of a submodule by two submodules of a module is never efficient. Thus $L \subseteq L_{1} \cup L_{2} \cup \ldots \cup L_{n}$ may be possibly an efficient covering only when $n=1$ or $n>2$ [6].

\section{Proposition}

Let $L \subseteq L_{1} \cup L_{2} \cup \ldots \cup L_{n}$ be an efficient covering consisting of submodules of an R-moduleM where $n>2$. If $\sqrt{\left[L_{j}: M\right]} \not \subset \sqrt{\left[L_{k}: M\right]}$ for every $j \neq k$, then no $L_{k}$ for 
$k=\{1, \ldots, n\}$ is a primary submodule of $\mathrm{M}$.

Proof. Since $L \subseteq L_{1} \cup L_{2} \cup \ldots \cup L_{n}$ is an efficient covering, $L=\left(L \cap L_{1}\right) \cup\left(L \cap L_{2}\right) \cup \ldots \cup\left(L \cap L_{n}\right) \quad$ is $\quad$ an efficient union. Hence for every $k \leq n$ there exists an element $e_{k} \in L-L_{k}$. Moreover, by Lemma (??), $\bigcap_{j \neq k}\left(L \cap L_{j}\right)=\bigcap_{j=1}^{n}\left(L \cap L_{j}\right) \subseteq\left(L \cap L_{k}\right)$ that is

$\bigcap_{j \neq k}\left(L \cap L_{j}\right) \subseteq\left(L \cap L_{k}\right)$. Now, for every $j \neq k$, by hypothesis, $\sqrt{\left[L_{j}: M\right]} \not \subset \sqrt{\left[L_{k}: M\right]}$ so that there exists $s_{j} \in \sqrt{\left[L_{j}: M\right]}$ but $s_{j} \notin \sqrt{\left[L_{k}: M\right]}$. Therefore there exists $\quad t_{j} \in Z^{+} \quad$ such that $\quad s_{j}^{t_{j}} M \subseteq L_{j}$. Let $t=\prod_{j \neq k} t_{j}=t_{1} . . t_{k-1} t_{k+1} . . t_{n}$. Suppose that some $L_{k}$ is a primary submodule so $\sqrt{\left[L_{k}: M\right]}$ is a prime ideal of $\mathrm{R}$. Let $s=\prod_{j \neq k} s_{j}=S_{1} \ldots s_{k-1} s_{k+1} \ldots s_{n}$. So $s^{t} M \subseteq L_{j}$ for every $j \neq k$. But $s \notin \sqrt{\left[L_{k}: M\right]}$. Consequently $s^{t} e_{k} \in\left(L \bigcap L_{j}\right)$ for every $j \neq k$. But $s^{t} e_{k} \notin\left(L \bigcap L_{k}\right)$. But this contradicts the fact that $\bigcap_{j \neq k}\left(L \cap L_{j}\right) \subseteq\left(L \cap L_{k}\right)$. Therefore

$L_{k}$ is not primary submodule.

\section{Theorem (The Primary Avoidance Theorem)}

Let $\mathrm{M}$ be an R-module $, L_{1}, L_{2}, \ldots, L_{n}$ a finite number of submodules of $\mathrm{M}$ and $L$ a submodule of $\mathrm{M}$ such that $L \subseteq L_{1} \cup L_{2} \cup \ldots \cup L_{n}$ assume that at most two of the $L_{i}{ }^{\prime} S$ are not primary submodules and that $\sqrt{\left[L_{j}: M\right]} \not \subset \sqrt{\left[L_{k}: M\right]}$ whenever $j \neq k$ then $L \subseteq L_{k}$ for some $\mathrm{k}$.

Proof. For the given covering $L \subseteq L_{1} \cup L_{2} \cup \ldots \cup L_{n}$, let $L \subseteq L_{i 1} \cup L_{i_{2}} \cup \ldots \cup L_{i_{m}}$ be an efficient reduction, then $1 \leq m \leq n$ and $m \neq 2$. If $m>2$ there exists at least one $L_{i j}$ which is primary. In view of proposition (1.4) this is impossible as $\sqrt{\left[L_{j}: M\right]} \not \subset \sqrt{\left[L_{k}: M\right]}$ if $j \neq k$. Hence $m=1$, thus $L \subseteq L_{k}$ for some k.

The condition $\sqrt{\left[L_{j}: M\right]} \not \subset \sqrt{\left[L_{k}: M\right]}$ if $j \neq k$ in the statement of the theorem is essential as is seen in example (1.2) If $\mathrm{N}$ is a p-compactly packed submodule of an R-moduleM, such that whenever $H \neq K$, then $\sqrt{[H: M]} \not \subset \sqrt{[L: M]}$ for every proper submodules $H$ and $L$ of $M$, then by the primary avoidance theorem, $\mathrm{N}$ is a strongly p-compactly packed submodule.

Recall that $\mathrm{J}(\mathrm{M})$ denotes the Jacobson Radical of $\mathrm{M}$ [4, p.55]. The following proposition shows that p-compactly packed modules which have $\mathrm{J}(\mathrm{M}) \neq \mathrm{M}$, satisfies a certain kind of ascending chain condition.

\section{Proposition}

Let $\mathrm{M}$ be a p-compactly packed R-module with $\mathrm{J}(\mathrm{M})$ $\neq \mathrm{M}$, then $\mathrm{M}$ satisfies the ascending chain condition for primary submodules.

Proof. $N_{1} \subseteq N_{2} \subseteq N_{3} \subseteq \ldots$ be an ascending chain of primary submodules of M. Let $N=\bigcup_{i} N_{i}$. We claim that $N \neq M$. In fact if $N=M$ and $H$ is a maximal submodule of $\mathrm{M}$ then $H \neq \bigcup_{i} N_{i}$, so there exists $n_{1}, n_{2}, \ldots, n_{k}$ such that $H \subseteq \bigcup_{i=1}^{k} N_{n_{i}}$, and since $N_{1} \subseteq N_{2} \subseteq N_{3} \subseteq \ldots$ is an ascending chain, so there exists $m \in\{1, \ldots, k\}$ such that $\bigcup_{i=1}^{k} N_{n_{i}}=N_{n_{m}}$ so $H \subseteq N_{n_{m}}$, then $H=N_{n_{m}}$, and consequently $M=\bigcup_{i} N_{i}=N_{n_{m}}$ which is a contradiction. So $\mathrm{N}$ is a proper submodule of $\mathrm{M}$, thus there exists $n_{1}, n_{2}, \ldots, n_{k}$ such that $N \subseteq \bigcup_{i=1}^{k} N_{n_{i}}$, and since $N_{1} \subseteq N_{2} \subseteq N_{3} \subseteq \ldots$ is an ascending chain, so there exists $m \in\{1, \ldots, k\}$ such that $\bigcup_{i=1}^{k} N_{n_{i}}=N_{n_{m}} \quad$ that $\quad$ is $\quad \bigcup_{i} N_{i} \subseteq N_{n_{m}} \quad$ so $N_{1} \subseteq N_{2} \subseteq N_{3} \subseteq \ldots N_{n_{m}}$. Therefore M satisfies the ascending chain condition on primary submodules.

Since finitely generated or multiplication module has a maximal submodule, the following corollary follows directly from the previous proposition.

\section{Corollary}

If $\mathrm{M}$ is a generated or multiplication p-compactly finitely module, then $M$ satisfies the ascending chain condition for primary submodules.

The following proposition and theorem give characterizations of strongly p-compactly packed modules. Recall that the primary radical of a submodule $\mathrm{N}$ of an $\mathrm{R}$ module $\mathrm{M}$, denoted by $\operatorname{prad}_{M}(N)$ is defined as the intersection of all primary submodules of $\mathrm{M}$ which contain $\mathrm{N}$. If there exists no primary submodule of $\mathrm{M}$ containing $\mathrm{N}$, we put $\operatorname{prad}_{M}(N)=\mathrm{M}[7]$.

A proper submodule $\mathrm{N}$ of an R-module $\mathrm{M}$ with $\operatorname{prad}_{M}(N)=\mathrm{N}$ will be called P-Radical Submodule [7].

\section{Proposition}

Let $\mathrm{M}$ be an $\mathrm{R}$-module. $\mathrm{M}$ is strongly $\mathrm{p}$-compactly packed if and only if every p-radical submodule of $\mathrm{M}$ is the primary radical of a cyclic submodule of it.

Proof. Let $\mathrm{N}$ be a p-radical submodule of $\mathrm{M}$ such that $\mathrm{N}$ is not the primaryradical of a cyclic submodule of it, thus for each $m \in N, N \neq \operatorname{prad}_{M}(\langle m\rangle)$. So there exists a primary submodule $L_{m} \supseteq\langle m\rangle$ but $N \not \subset L_{m}$.

Thus

$N=\cup_{m \in N}\langle m\rangle \subseteq \cup_{m \in N} L_{m}$ for $\langle m\rangle \subseteq L_{m} \not \subset N$. That is $L_{m} \not \subset N$ for each $m \in N$.

This contradicts that $\mathrm{M}$ is strongly p-compactly packed 
module. Conversely, let $N \subseteq \bigcup_{\alpha \in \Lambda} N_{\alpha}$ where $N_{\alpha}$ is a primary submodule of

M for each $\alpha \in \lambda$ and $N=\operatorname{prad}_{M}(\langle m\rangle)$ for some $m \in N$. Since $m \in N, m \in \bigcup_{\alpha \in \Lambda} N_{\alpha}$, so there exists

$\beta \in \Lambda \quad$ such that $m \in N_{\beta} \quad, \quad$ hence

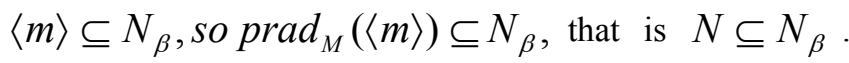
Therefore $\mathrm{M}$ is a strongly $\mathrm{p}$-compactly packed module.

\section{Theorem}

Let $\mathrm{M}$ be an R-module. The following statements are equivalent:-

1) $\mathrm{M}$ is a strongly p-compactly packed module.

2) For every proper submodule $N$ of $M$, there exists $\quad m \in N$ such that $\operatorname{prad}_{M}(N)=\operatorname{prad}_{M}(\langle m\rangle)$.

3) For every proper submodule $\mathrm{N}$ of $\mathrm{M}$, if $\left\{N_{\alpha}\right\}_{\alpha \in \Lambda}$ is a family of submodules of $\mathrm{M}$, such that

$N \subseteq \cup N_{\alpha}$, then there exists $\beta \in \Lambda$ such that $N \subseteq \underset{\alpha \in \Lambda}{\subseteq} \operatorname{prad}_{M}\left(N_{\beta}\right)$.

4) For every proper submodule $\mathrm{N}$ of $\mathrm{M}$, if $\left\{N_{\alpha}\right\}_{\alpha \in \Lambda}$ is a family of p-radical submodules of $\mathrm{M}$, with $N \subseteq\left\{N_{\alpha}\right\}_{\alpha \in \Lambda}$ there exists $\beta \in \Lambda$ such that $N \subseteq N_{\beta}$.

Proof. $(1) \Rightarrow(2)$ : By the same argument of the proof of (1.8).

$(2) \Rightarrow(3)$ : Let $\mathrm{N}$ be a proper submodule of $\mathrm{M}$ and $\left\{N_{\alpha}\right\}_{\alpha \in \Lambda}$ be a family of submodules of $\mathrm{M}$, such that $N \subseteq \cup_{\alpha \in \Lambda} N_{\alpha}$. By (2) there exists $m \in N$ such that $\operatorname{prad}_{M}(N)=\operatorname{prad}_{M}(\langle m\rangle)$. Since $m \in \bigcup_{\alpha \in \Lambda} N_{\alpha}$, it follows

that there exists $\beta \in \Lambda$ such that $m \in N_{\beta}$ hence

$\langle m\rangle \subseteq N_{\beta}$, so $N \subseteq \operatorname{prad}_{M}(N)=\operatorname{prad}_{M}(\langle m\rangle) \subseteq \operatorname{prad}_{M}\left(N_{\beta}\right)$

$(3) \Rightarrow(4)$ : It follows directly from the definition of Pradical submodule $\mathrm{N}$.

(4) $\Rightarrow(1)$ : It is trivial.

In what follows we give a proposition which gives information about a strongly p-compactly packed module with $J(M) \neq M$.

\section{Proposition}

Let $\mathrm{M}$ be a strongly p-compactly packed R-module such that $J(M) \neq M$. Then $\mathrm{M}$ satisfies the ascending chain condition for P-radical submodules.

Proof. Let $N_{1} \subseteq N_{2} \subseteq N_{3} \subseteq \ldots$ be an ascending chain of primary p-radical submodules of $M$. Let
$L=\bigcup_{i} N_{i}$, then $\mathrm{L}$ is a submodule of $\mathrm{M}$. We claim that $\mathrm{L}$ is a proper submodule of $\mathrm{M}$. In fact if $L=M$ and $\mathrm{H}$ a maximal submodule of $\mathrm{M}$, so $H \not \subset \bigcup_{i} N_{i}$ then by theorem ((1.9)(iv)) there exists $\mathrm{j}$ such that $H \subseteq N_{j}$ and since $\mathrm{H}$ is a maximal submodule $H=N_{j}$ and this implies $\bigcup_{i} N_{i} \subseteq N_{j}$ that is $M \subseteq N_{j}$ which is a contradiction. So $\mathrm{L}$ is a proper submodule of $\mathrm{M}$ and by theorem (1.9) there exists j such that $L \subseteq N_{j} \quad$ so $N_{1} \subseteq N_{2} \subseteq N_{3} \subseteq \ldots N_{j}$ that is $\mathrm{M}$ satisfies the ascending chain condition for p-radical submodules.

The following is an immediate consequence of proposition (1.10).

\section{Corollary}

Let $\mathrm{M}$ be a finitely generated or multiplication strongly $\mathrm{p}$ compactly packed R-module, then $\mathrm{M}$ satisfies the ascending chain condition for $\mathrm{p}$-radical submodules.

Recall that an R-module $\mathrm{M}$ is called Bezout Module if every finitely generated submodule of $\mathrm{M}$ is cyclic.

In the following proposition we give a condition for the converse of proposition (1.10) to hold.

\section{Proposition}

Let $\mathrm{M}$ be a Bezout $\mathrm{R}$-module. If $\mathrm{M}$ satisfies the ascending chain condition for P-radical submodules, then $\mathrm{M}$ is strongly p-compactly packed module.

Proof. Let $\mathrm{N}$ be a proper submodule of $\mathrm{M}$, it is easy to show that there exists a finitely generated submodule $\mathrm{L}$ of $\mathrm{N}$ such that $\operatorname{prad}_{M}(N)=\operatorname{prad}_{M}(L)$ But $\mathrm{M}$ is Bezout module so $\mathrm{L}$ is a cyclic submodule, there exists $m \in L$, such that $L=\langle m\rangle$, this implies $m \in N$ and $\operatorname{prad}_{M}(N)=\operatorname{prad}_{M}(\langle m\rangle)$ therefore by theorem (1.9), $\mathrm{M}$ is a strongly p-compactly packed module.

Now, we give a characterization of a strongly pcompactly packed finitely generated or multiplication module.

\section{Proposition}

Let $\mathrm{M}$ be a multiplication or finitely generated R-module. If we have one of the following:

1) $\mathrm{M}$ is a cyclic module .

2) $\mathrm{M}$ is a Bezout module .

3) $\mathrm{R}$ is a Bezout ring .

Then $\mathrm{M}$ is a strongly $\mathrm{p}$-compactly packed module if and only if every primary submodule of $\mathrm{M}$ is a strongly $\mathrm{p}$ compactly packed submodule.

Proof. Suppose that every primary submodule of $M$ is strongly $\mathrm{p}$-compactly packed. Let $\mathrm{N}$ be a proper submodule of $\mathrm{M}$ such that $N \subseteq \bigcup_{\alpha \in \Lambda} N_{\alpha}$ where $N_{\alpha}$ is a primary submodule of $\mathrm{M}$ for each $\alpha \in \Lambda$. Assume $\bigcup_{\alpha \in \Lambda} N_{\alpha}=M$. Then $\mathrm{L}$ is strongly p-compactly packed and since $N \subseteq L \subset M=\bigcup_{\alpha \in \Lambda} N_{\alpha}$, so there exists $\beta \in \Lambda$ 
such that $L \subseteq N_{\beta}$, hence $N \subseteq L \subseteq N_{\beta}$. Now if $\cup N_{\alpha} \neq M \quad, \quad$ let $\quad S^{*}=M-\bigcup N_{\alpha} \quad$ and $S=R-\bigcup_{\alpha \in \Lambda} \sqrt{\left[N_{\alpha}: M\right]}$

so $S^{*}$ is an S-closed subset of $\mathrm{M}$ and since $N \subseteq \bigcup_{\alpha \in \Lambda} N_{\alpha}$ it follows $N \subseteq M-S^{*}$,so there exists a submodule $\mathrm{L}$

Maximal in $M-S^{*}$ and contains $\mathrm{N}[1, \mathrm{p} .75], \mathrm{L}$ is a prime [1, p. 61], so primary submodule, but $L \subseteq \bigcup_{\alpha \in \Lambda} N_{\alpha}$ (because $L \subseteq M-S^{*}$ ) so there exists $\beta \in \Lambda$ such that $L \subseteq N_{\beta}$, hence $N \subseteq L \subseteq N_{\beta}$ Therefore $\mathrm{M}$ is a strongly p-compactly packed module. The converse is trivial.

In the remainder of this section we shall investigate the relation between the strongly p-compactly packed modules, p-compactly packed modules and the modules of fractions.

Our next result has some interest in itself.

\section{Lemma}

Let $\mathrm{M}$ be an R-module and $\mathrm{S}$ a multiplicatively closed set in $\mathrm{R}$. If $\mathrm{W}$ is a primary submodule of the $R_{S}$-module $M_{S}$, then $\phi^{-1}(W)$ is a primary submodule of $\mathrm{M}$.

Proof. Suppose that $\mathrm{W}$ is a primary submodule of $M_{S}$. First to show that is proper submodule of $\mathrm{M}$, it is sufficient to show $\left[\phi^{-1}: M\right] \cap S=\varphi$. Suppose $r \in\left[\phi^{-1}: M\right] \cap S$, thus $r \in S$ and $r m \in \phi^{-1}(W)$ for all $m \in M, \phi(r m)=\frac{r m}{1} \in W$, for all $m \in M$. Let $\frac{a}{t} \in M_{S}$, so $\frac{a}{t}=\frac{r a}{r t}=\frac{r a}{1} \cdot \frac{1}{r t} \in W$, thus $M_{S} \subseteq W$ which is contradiction.

Now to show $\phi^{-1}(W)$ is primary submodule, let $r \in R$, $m \in M \quad$ such that $r m \in \phi^{-1}(W) \quad$ so $\quad \phi(r m) \in W$, $\frac{r m}{1}=\frac{r}{1} \cdot \frac{m}{1} \in W$ but $\mathrm{W}$ is primary submodule of $M_{S}$, hence either $\frac{m}{1} \in W \quad$ or $\quad \phi(m)=\frac{m}{1} \in W \quad$ this implies $m \in \phi^{-1}(W)$ or $\frac{r}{1} \in \sqrt{\left[W: M_{S}\right]}$ so there exists $n \in Z^{+}$such that $\frac{r^{n}}{1} \cdot \frac{m}{s} \in W$ for all $\frac{m}{s} \in M_{S}$.

Therefore $\phi\left(r^{n} m\right)=\frac{r^{n} m}{1}=\frac{r^{n} m s}{s}=\frac{r^{n} m}{s} \cdot \frac{s}{1} \in W \quad$, hence $r^{n} m \in \phi^{-1}(W)$ for all $m \in M$, thus $r \in \sqrt{\left[\phi^{-1}(W): M\right]}$, therefore $\phi^{-1}(W)$ is primary.

Now, we look at the relation between strongly pcompactly packed module $\mathrm{M}$, and the module of fractions $M_{S}$.

\section{Proposition}

Let $\mathrm{M}$ be an R-module and $\mathrm{S}$ a multiplicatively closed set in $\mathrm{R}$. If $\mathrm{M}$ is strongly p-compactly packed R-module then $M_{S}$ is strongly p-compactly packed $R_{S}$-module.

Proof. Suppose $H \subseteq \bigcup_{\alpha \in \Lambda} W_{\alpha}$ where $\mathrm{H}$ is a proper submodule of $M_{S}$ and $W_{\alpha}$ is a primary submodule of $M_{S}$ for each $\alpha \in \Lambda$. Hence $\phi^{-1}(H) \subseteq \phi^{-1}\left(\bigcup_{\alpha \in \Lambda} W_{\alpha}\right)$. So $\phi^{-1}(H) \subseteq \bigcup_{\alpha \in \Lambda} \phi^{-1}\left(W_{\alpha}\right)$ By Lemma (1.14) $\phi^{-1}\left(W_{\alpha}\right)$ is a primary submodule of $\mathrm{M}$, there exists $\beta \in \Lambda$ such that $\phi^{-1}(H) \subseteq \phi^{-1}\left(W_{\beta}\right)$ hence $\left(\phi^{-1}(H)\right)_{S} \subseteq\left(\phi^{-1}\left(W_{\beta}\right)\right)_{S}$. We will show that $\left(\phi^{-1}(K)\right)_{S}=K$ for every submodule $\mathrm{K}$ of $M_{S}$. Let $\frac{x}{S} \in\left(\phi^{-1}(K)\right)_{S}$ where $x \in \phi^{-1}(K)$ and $s \in S$. So $\phi(x) \in K$, that is $\frac{x}{1} \in K$, hence $\frac{x}{1} \cdot \frac{1}{s}=\frac{x}{S} \in K$, so $\left(\phi^{-1}(K)\right)_{S} \subseteq K$. Now let $\frac{x}{s} \in K$, thus $\frac{x}{s} \cdot \frac{s}{1} \in K$, hence $\frac{x}{1} \in K$ that is $\phi(x) \in K$ so $x \in \phi^{-1}(K)$, thus $\frac{x}{s} \in\left(\phi^{-1}(K)\right)_{S}$ therefore $K \subseteq\left(\phi^{-1}(K)\right)_{S}$, consequently $K=\left(\phi^{-1}(K)\right)_{S}$ for all $K \subseteq M_{S}$. It follows $H \subseteq W_{\beta}$. Hence $M_{S}$ is a strongly p-compactly packed module.

Turning now to the relation between p-compactly packed module $\mathrm{M}$ and the module of fractions $M_{S}$.

\section{Proposition}

Let $\mathrm{M}$ be an R-module and $\mathrm{S}$ a multiplicatively closed set in $\mathrm{R}$. If $\mathrm{M}$ is $\mathrm{p}$-compactly packed R-module then $M_{S}$ is $\mathrm{p}$ compactly packed $R_{S}$-module.

Proof. Let $H \subseteq \bigcup_{\alpha \in \Lambda} W_{\alpha} \mathrm{H}$ is a proper submodule of $M_{S}$ and $W_{\alpha}$ is a primary submodule of $M_{S}$ for each $\alpha \in \Lambda$.

Hence $\quad \phi^{-1}(H) \subseteq \phi^{-1}\left(\bigcup_{a \in \Lambda} W_{\alpha}\right)$ So $\phi^{-1}(H) \subseteq \bigcup_{\alpha \in \Lambda} \phi^{-1}\left(W_{\alpha}\right)$ By Lemma (1.14) $\phi^{-1}\left(W_{\alpha}\right)$ is a primary submodule of $\mathrm{M}$,

there exists $\alpha_{1}, \alpha_{2}, \ldots, \alpha_{n} \in \Lambda \quad$ such that $\phi^{-1}(H) \subseteq \bigcup_{i=1}^{n} \phi^{-1}\left(W_{\alpha i}\right)$ hence $\left(\phi^{-1}(H)\right)_{S} \subseteq \bigcup_{i=1}^{n}\left(\left(\phi^{-1}\left(W_{\beta}\right)\right)\right)_{S}=\bigcup_{i=1}^{n}\left(\phi^{-1}\left(W_{\alpha_{i}}\right)\right)_{S}$.

Now, as in the proof of proposition (1.15), $H \subseteq \bigcup_{\alpha \in \Lambda} W_{\alpha}$. Therefore $M_{S}$ is p-compactly packed module.

The converses of the last two propositions are not true in general as is seen in the following example:

\section{Example}

Let $\mathrm{X}$ be an infinite set. Let $\mathrm{R}$ be the ring $(P(X), \Delta, \cap)$ which is a Bolean ring so it is regular.

Let $\mathrm{T}=\{\mathrm{H} \mid \mathrm{H}$ is a finite subset of $\mathrm{X}\}$, so $\mathrm{T}$ is nonmaximal ideal of $\mathrm{P}(\mathrm{X})$, and for any $H \in T$ we have $\langle H\rangle$ is a radical ideal, since every proper ideal in a regular ring is radical ideal. This implies that $\langle H\rangle=\bigcap\{\mathrm{P} \mid \mathrm{P}$ is a prime ideal contains $\mathrm{H}\}$. It is easy to show that every primary ideal $\mathrm{L}$ of $\mathrm{P}(\mathrm{X})$ is prime. This implies that $\operatorname{prad}_{P(X)}(H)=\langle H\rangle$, since $T \not \subset\langle H\rangle$ for all $H \in T$, that is $T \not \subset \operatorname{prad}_{P(X)}(\langle H\rangle)$ for all $H \in T$ so there exists primary ideal $P_{H}$ such that $P_{H} \supseteq\langle H\rangle$ 
but $T \not \subset P_{H}$. Since $T=\bigcup_{H \in T}\langle H\rangle \subseteq \bigcup_{H \in T} P_{H}$ So $\mathrm{T}$ is not p-compactly packed submodule. So $\mathrm{P}(\mathrm{X})$ is not p-compactly packed module.

On the other hand, for any maximal ideal $\mathrm{P}$ of $\mathrm{R}, R_{P}$ is a field because $\mathrm{R}$ is a regular ring, so $R_{P}$ is p-compactly packed $R_{P}$-module.

\section{REFERENCES}

[1] A. Z. Ani, "Compactly Packed Modules and Coprimely Packed Modules," M.Sc. Thesis, Baghdad University, College of Science, 1996.
[2] V. Erdo gdu, "Coprimely Packed Rings," J. of Number Theory, vol. 28, pp.1-5, 1989

[3] C. Gottlieb, "On Finite Unions of Ideals and Cosets," Comm. Algebra, vol. 22, 3087-3097, 1994.

[4] M. D. Larsen and P. J. McCarlthy, "Multiplication Theory of Ideals," Academic Press, New York, 1971.

[5] C. P. Lu, "Union of Prime Submodules, Houston J., Math," vol. 23, pp. 203-213, 1997

[6] N. McCoy, "A Note on Finite Unions of Ideals and Subgroups," in Proc. Amer. Math. Soc, vol. 8, pp.633-637, 1957.

[7] J. M. A. Lamis, "The Primary Radical of a submodule, Advances in Pure Mathematics," 2012. 